\title{
COMMENTS ON A FULL QUANTIZATION OF THE TORUS
}

\author{
J.M. VELHINHO \\ Universidade do Algarve, U.C.E.H., Área Departamental de Física. \\ Campus de Gambelas, 8000 Faro, Portugal
}

\begin{abstract}
Gotay showed that a representation of the whole Poisson algebra of the torus given by geometric quantization is irreducible with respect to the most natural overcomplete set of observables. We study this representation and argue that it cannot be considered as physically acceptable. In particular, classically bounded observables are quantized by operators with unbounded spectrum. Effectively, the latter amounts to lifting the constraints that compactify both directions in the torus.
\end{abstract}

\section{Introduction}

There are still some open questions as to what is precisely meant by quantization of a general symplectic manifold. Two interconnected problems affect Dirac's original formulation: (a) which Poisson subalgebra should one choose to impose the Dirac quantum condition "Poisson bracket goes to commutator"; (b) which extra conditions should be required in order to guarantee that the quantum representation is physically meaningful.

Although no general result was ever proved, it is a common belief that a physically acceptable quantization of the whole Poisson algebra cannot be found, for any symplectic manifold (see below). One way to circumvent the obstruction to a full quantization consists in looking for quantum representations of some convenient proper subalgebra $\mathcal{S}$ of the whole Poisson algebra. It is generally acknowledged that the subalgebra $\mathcal{S}$ to be quantized must be (kinematically) complete, in the sense that it should contain a complete set of classical observables. However, the precise definition of a complete set of classical observables $\mathcal{B}, \mathcal{B} \subset \mathcal{S}$, is not so consensual. In Ref. 1, A.A. Kirillov defines a set of observables $\mathcal{B}$ to be complete if the only observables which (Poisson) commute with every element of $\mathcal{B}$ are the constant functions (this, in particular, means that the functions in $\mathcal{B}$ separate points locally almost everywhere on the phase space). A stronger condition (in the sense that it guarantees local separation of points everywhere) is required by M.J. Gotay, H.B. Grundling and G.M. Tuynman, reading: the Hamiltonian vector fields 
associated with the functions in $\mathcal{B}$ should generate the tangent space everywhere in phase space. 1 Notice that, as the authors acknowledge, none of the above two conditions guarantees global separation of points. The latter, we think, must be imposed in order to make sure that one is quantizing the initial phase space and not its quotient by the relations defined by considering two points equivalent if they are not separated by the functions in the set $\mathcal{B}$. A similar strong notion of completeness is favored by A. Ashtekar, which requires that the subalgebra $\mathcal{S}$ should be "large enough" so that any (sufficiently regular) function on the phase space can be represented as (possibly a limit of) a sum of products of elements of $\mathcal{S}$.

In order to avoid the appearance at the quantum level of spurious degrees of freedom, one imposes, in one way or another, irreducibility conditions on the quantum representations (see Ref. 2 for a discussion). Following Ref. 2 (see also Ref. 1), we will concentrate on an explicit irreducibility requirement: the quantum Hilbert space should be irreducible with respect to the action of all operators representing a complete set of classical observables.

Many examples of representations of "large" Poisson subalgebras (possibly the whole algebra) which do not comply with irreducibility are known; for instance the so called prequantizations obtained through the methods of Geometric Quantization. 1 It is generally acknowledged that these examples are manifestations of a general obstruction to quantization: the widely accepted "no-go conjecture" claims, in essence, that a physically relevant quantization of a full Poisson algebra is unattainable, due to the impossibility of satisfying simultaneously the Dirac quantum condition on the whole algebra and the irreducibility requirement. The first rigorous "no-go" result goes back to the works of Groenewold and Van Hove (see Ref. 2 and references therein) and states that it is impossible to fulfill the Dirac quantum condition for the whole Poisson algebra on $\mathbb{R}^{2}$ while keeping irreducibility on the level of the Poisson subalgebra generated by $q$ and $p$ (the Heisenberg algebra $\mathrm{h}(2)=\operatorname{span}\{1, q, p\}$ of inhomogeneous degree one polynomials in $q$ and $p$ ). This is the famous "no-go theorem" for $\mathbb{R}^{2}$ (which is readily generalizable to $\mathbb{R}^{2 n}$ ). Recently, similar no-go results also based on irreducibility were proven for the sphere $S^{2}$ and the cylinder $T^{*} S^{1}$.

The standard quantization procedure requires therefore the selection of a preferred subalgebra $\mathcal{S}$ of classical observables. This special subalgebra must be complete and should be such that irreducibility can be achieved. It is also assumed that the constant function 1 belongs to $\mathcal{S}$ and should be mapped to the identity operator (see Ref. 1 and Ref. 2). Clearly, no generality is lost in assuming that $1 \in \mathcal{S}$, for given a subalgebra $\mathcal{S}$ which does not contain 1, every acceptable quantization map can be extended just by assigning the identity operator to the function 1 . Notice also that the above condition on the quantization of 1 is independent from irreducibility and is of a quite different nature. The " 1 goes to 1 " condition is required to enforce the correspondence between the classical value attained by the constant observable and its quantum spectrum.

Unlike the above outlined conditions, when considering general observables there 
seems to be no major agreement on the important subject of the relation between the classical range of values attained and the quantum spectrum of the observable. This is closely related with the issue of preservation of the classical multiplicative structure. Along with the Poisson structure, pointwise multiplication of observables is a fundamental aspect of the classical theory and one certainly would like to see it reflected at the quantum level. The question is to what extent should the multiplicative structure be preserved upon quantization, given that the Poisson and the multiplicative structure are not fully compatible at the quantum level (see Ref. 2). Due to this fact, some authors take the point of view that quantization concerns the preservation of Poisson relations only, thus choosing not to impose a priori any further conditions on quantization (besides irreducibility and "1 goes to 1") 1 On the other hand, one can find in the literature explicit concern about the relations between the classical range (which we will call classical spectrum) and the quantum spectrum (Ref. 6) and also the requirement that relations among preferred classical observables coming from the multiplicative structure should be implemented in the quantum theory through appropriate anti-commutation relations (Ref. 3, Ref. 7).

In our opinion, multiplicative algebraic relations among preferred classical observables are fundamental and should be carried over to the quantum theory. Let us assume that a preferred Poisson subalgebra $\mathcal{S}$ of real valued classical observables has been chosen. Although $\mathcal{S}$ is, in general, not closed under multiplication, it still reflects the multiplicative structure and also the global topology of phase space. Let us consider first $\mathbb{R}^{2}$. In this case, global, canonically conjugate, coordinates $q$ and $p$ exist and the algebra $\mathrm{h}(2)$ generated by these observables is complete. Of course, no relations can be found among the generators of $\mathrm{h}(2)$, since $q$ and $p$ are independent, linearly or otherwise. A different situation occurs if the Poisson subalgebra $\operatorname{span}\left\{1, q, q^{2}, p, p^{2}\right\}$ of polynomials of degree no greater than two in $q$ and $p$ is chosen. In this case, multiplication relates, for instance, $q$ and $q^{2}$, encoding the obvious fact that $q^{2}$ is a positive valued observable whose values in every state are completely determined by the observed values of $q$.

Whereas in $\mathbb{R}^{2}$ (or $\mathbb{R}^{2 n}$ ) it is always possible to choose a complete Poisson subalgebra which is free of relations, this is no longer the case when the phase space has a globally non-trivial topology. In the absence of a global chart, a set of everywhere defined functions which separates points is necessarily overcomplete, meaning that the number of functions in the set exceeds the dimensionality of the phase space. Clearly, the functions in a overcomplete set cannot be all independent and therefore relations among them must appear. A standard example of this situation is provided by the cylinder $T^{*} S^{1}$, the phase space of a particle moving on a circle. Since the angular variable $\theta$ on $S^{1}$ is not globally defined, the best one can do is to work with the functions $\sin \theta, \cos \theta$ and $p$, where $p$ is the conjugate momentum of $\theta$. In general, the global non-triviality of the phase space will therefore manifest itself in every admissible preferred subalgebra $\mathcal{S}$, in the form of a set of functional relations among elements of $\mathcal{S}$. These relations carry crucial information about the interdependence of observables, their spectrum and the global aspects of the phase 
space.

In order to illustrate the problems that might occur if algebraic relations are neglected in the quantization process, let us consider first the above example of the algebra $\operatorname{span}\left\{1, q, q^{2}, p, p^{2}\right\}$ in $\mathbb{R}^{2}$, which includes both $q$ and $q^{2}$. Upon quantization, one then has two well defined commuting observables $\hat{q}$ and $\widehat{q^{2}}$. Let us assume that $\widehat{q^{2}}$ is not equal to $(\hat{q})^{2}$. Then it is possible that the operator $\widehat{q^{2}}$, the quantum analog of $q^{2}$, is not positive, leading to states with negative expectation values. In contrast, even though $(\hat{q})^{2}$ is a well defined operator which relates to $\hat{q}$ as expected, it is not clear what meaning should one assign to this operator, since the quantum analog of $q^{2}$ was already determined.

One can illustrate further the importance of algebraic relations by means of an example of a globally non-trivial phase space. $T^{*} S^{1}$. A natural choice for $\mathcal{S}$ in this case would be the vector space spanned by the functions $\{1, \sin \theta, \cos \theta, p\}$. Suppose one is given an irreducible Lie-representation $\rho$ of $\mathcal{S}$ such that the relation

$$
(\rho(\sin \theta))^{2}+(\rho(\cos \theta))^{2}=\mathbf{1}
$$

does not hold. It is then clear that $\theta$ looses its meaning as an angular variable, and that one runs into serious problems with the physical interpretation of the simultaneously measured values of $\rho(\sin \theta)$ and $\rho(\cos \theta)$. The natural thing to do is to demand (11) to be satisfied (this condition is, of course, indeed satisfied in the usual quantization on $L^{2}\left(S^{1}, d \theta\right)$, where $\hat{p}=-\mathrm{i} \hbar \frac{d}{d \theta}$ and $\widehat{\sin \theta}, \widehat{\cos \theta}$ act by multiplication). This is the point of view taken in Ref. 3 and Ref. 7 , where the authors propose the exact implementation in the quantum theory of algebraic relations among (Poisson) commuting elements of the special classical subalgebra $\mathcal{S}$. Also proposed is the following natural rule to handle relations among non-commuting elements of $\mathcal{S}$ : if $F_{1}, F_{2}, \ldots F_{m}$ and $G$ are elements of $\mathcal{S}$ such that $F_{1} F_{2} \ldots F_{m}=G$ then one requires $\hat{F}_{(1} \ldots \hat{F}_{m)}=\hat{G}$ to be satisfied for the corresponding quantum operators, where the bracket denotes symmetrization (this rule generalizes in the natural way to linear combinations of products) 目团

The above considerations indicate that one runs into serious ambiguities if relations among classical observables included in the preferred subalgebra $\mathcal{S}$ are broken at the quantum level. In particular, if relations among Poisson commuting (unambiguously quantized) classical observables are ignored, one allows the appearance of quantum operators whose spectrum bears no relation with the classical range, thus making the physical interpretation of the theory quite problematic. A well defined procedure to handle algebraic relations is therefore an important ingredient of quantization. An important physical example is provided by non-Abelian theories of connections with a compact gauge group, where the gauge invariant configuration space is a globally non-trivial infinite-dimensional manifold. 10 In this case, a convenient (over)complete set of variables is provided by the Wilson loop variables, but one must deal with the algebraic relations among them (the so-called Mandelstam identities); this is just the price to pay for working with gauge invariant functions 
(see Ref. 3 and references therein).

A new question which arises in this framework consists in verifying the compatibility between the above rule to handle algebraic relations, on one hand, and the preservation of Poisson structure and irreducibility, on the other. Although this question seems to be a difficult one, in general, one might gain some insight through the analysis of simple cases. Consider again $\mathbb{R}^{2}$, and choose $\mathcal{S}$ to be the Poisson subalgebra of polynomials of degree no greater than two in $q$ and $p$. It is a trivial matter to verify that the Schrödinger quantization of $h(2)$ extends to this new algebra by the following new assignments:

$$
\widehat{q^{2}}=q^{2}, \quad \widehat{q p}=-\mathrm{i} \hbar\left(q \frac{d}{d q}+1 / 2\right), \quad \widehat{p^{2}}=-\hbar^{2} \frac{d^{2}}{d q^{2}} .
$$

Since this is an extension of the representation of $h(2)$, irreducibility is guaranteed. On the other hand, it is clear that $\widehat{q^{2}}=(\hat{q})^{2}, \widehat{p^{2}}=(\hat{p})^{2}$ and $2 \widehat{q p}=\hat{q} \hat{p}+\hat{p} \hat{q}$. Thus, in this particular case, one finds no incompatibility. The same happens with the extension of the Schrödinger representation of $\mathrm{h}(2)$ to the subalgebra of $C^{\infty}\left(\mathbb{R}^{2}\right)$ which is made of inhomogeneous degree one polynomials in $p$, with arbitrary functions of $q$ as coefficients. Also, for $T^{*} S^{1}$, the above mentioned quantization of $\operatorname{span}\{1, \sin \theta, \cos \theta, p\}$ is extendible to functions linear in $p$ in a similar fashion, again fulfilling both the irreducibility and preservation of functional relations requirements (see Ref. 2 for a detailed discussion of these quantizations, as well as obstructions to further extension). A less trivial and very interesting case is provided by the sphere $S^{2}$. Since the area has the same dimensions as the Planck constant $h$, one can write the canonical area form $\omega$ on $S^{2}$ as

$$
\omega=(K h / 4 \pi) \sin \theta d \theta d \phi,
$$

where $\theta, \phi$ are the usual angular variables and $K$ is a dimensionless constant which determines the total area. The linearly independent functions

$$
f_{1}=K / 2 \sin \theta \cos \phi, f_{2}=K / 2 \sin \theta \sin \phi, f_{3}=K / 2 \cos \theta
$$

obviously separate points and are closed under the Poisson bracket:

$$
\left\{f_{i}, f_{j}\right\}=\frac{1}{\hbar} \epsilon_{i j k} f_{k} .
$$

The linear space spanned by $f_{1}, f_{2}, f_{3}$ and the constant function 1 is thus a natural choice for the special subalgebra $\mathcal{S}$. One has, of course, the following relation among elements of $\mathcal{S}$ :

$$
\sum_{i} f_{i}^{2}-(K / 2)^{2}=0 .
$$

A quantization of $\mathcal{S}$ is given by a representation of $s u(2)$. The irreducible representations of $\mathcal{S}$ are thus well known, indexed by integers or half-integers $j$, and satisfy

$$
\sum_{i}\left(\hat{f}_{i}\right)^{2}-j(j+1) \mathbf{1}=0 .
$$


Equation (4) strongly resembles (3), thus suggesting that (4) should be properly interpreted as the quantum counterpart of (3). Suppose first one ignores (3), thus requiring irreducibility only as an extra condition on quantization. One then gets a countable family of equally good candidates to the quantum theory associated with the sphere of area $K h$. Uniqueness is obviously not achieved. It is only when one demands further the preservation of relation (3) that a unique representation is selected. Clearly, the relation (3) can only be exactly preserved for those values of $K$ which equal $\sqrt{2 j(2 j+2)}$ for some integer or half-integer $j$. Thus, the point of view of exact implementation of functional relations leads to the conclusion that a proper quantum theory for the sphere can only be defined for a discrete set of values of the area. This "quantization" of the area should not come as a surprise: it is known that $S^{2}$ is the reduced phase space associated with a system of two identical uncoupled harmonic oscillators constrained to have a definite value of the total energy. Since, from standard Quantum Mechanics, the allowed values of the energy of such a system form a discrete set, one immediately obtains the quantization of the area, because the area of the sphere is determined by the value of energy (see Ref. 7 for a quantization of the sphere along these lines).

In the above examples it was possible to preserve the functional relations within the special subalgebra $\mathcal{S}$ in a way which was not incompatible with the irreducibility requirement. In the case of the sphere, although the irreducible representations automatically fulfill a relation similar to the classical one, the explicit requirement of exact matching is necessary to obtain a unique quantization. The additional condition selects from among the set of all irreducible representations the one with the dimensionality corresponding to the given area of the classical phase space. In particular, one can see that the spectrum of the quantum operators $\hat{f}_{i}$ becomes a subset of the range of the corresponding classical observables. Notice also that for large quantum numbers $j$ the relation between area of phase space and dimensionality of Hilbert space is just what one might expect from semi-classical arguments, since both dimensionality and the ratio of the area to the Planck constant $h$ approach the integer $2 j$.

\section{The Torus}

Recently M.J. Gotay et al. reexamined the concept of quantization and looked for obstructions of the Groenewold-Van Hove type for manifolds other than $\mathbb{R}^{2 n}$. Their work is presented in a series of papers which, besides formulating a general "no-go theorem", aims at giving a precise meaning to quantization and finding maximal quantizable algebras. prequantization: modulo technicalities regarding domains of operators, prequantization is a linear map from some Poisson subalgebra (possibly the whole algebra) to self-adjoint operators such that the "Poisson bracket goes to commutator" rule is satisfied and the constant function 1 is mapped to the identity operator. To pass from a prequantization to a quantization in a general case, the authors of Ref. 2 propose an approach based on the Poisson structure and on irreducibility only: no 
relations among observables coming from the multiplicative algebra structure are imposed a priori. They look for a (minimal) "basic" set carrying the kinematical information and demand this set to be irreducibly represented.

As a result of their work, Gotay et al. proposed a weaker than expected "no-go theorem", on account of a new result which the authors interpreted as a genuine full quantization.2.10 The authors proved the existence of obstructions to quantization for the sphere $S^{2}$ and the cylinder $T^{*} S^{1}$. 4 - These results points in the same direction as Groenewold and Van Hove's and thus could be considered as further evidence in favor of a strong "no-go theorem". However, the authors found no obstruction to the quantization of the torus $T^{2}$. In fact, it was shown that a prequantization of the whole Poisson algebra of the torus produced via geometric quantization (see Ref. 1) is in fact a quantization in the sense defined in Ref. 2 and outlined above, meaning that a basic set is irreducibly represented. Thus, a quantization of the whole Poisson algebra of the torus is claimed.210 Although the proven irreducibility is an interesting result, we disagree that the full prequantization of $T^{2}$ can be considered a physically acceptable quantization of the torus. We discuss this point next, starting with a brief review of the results presented in Ref. 10 (in what follows $h$ is Planck's constant and $N$ a non-zero integer).

One realizes the torus $T^{2}$ of area $N h$ as $\mathbb{R}^{2} / \mathbb{Z}^{2}$ with symplectic form

$$
\omega_{N}=N h d x \wedge d y
$$

and identifies the Poisson algebra $C^{\infty}\left(T^{2}\right)$ with the periodic $C^{\infty}$-functions $f$ on $\mathbb{R}^{2}$

$$
f(x+m, y+n)=f(x, y), \quad \forall m, n \in \mathbb{Z} .
$$

The prequantum Hilbert space is given by the rules of geometric quantization; it is made of sections of a complex line bundle over $T^{2}$. In Ref. 1 it is shown that the prequantum Hilbert space can be realized as the completion of the space $\Gamma_{N}$ of complex $C^{\infty}$-functions $\phi$ on $\mathbb{R}^{2}$ satisfying

$$
\phi(x+m, y+n)=e^{2 \pi i N m y} \phi(x, y), \quad \forall m, n \in \mathbb{Z},
$$

with inner product

$$
<\phi, \phi^{\prime}>=\int_{[0,1) \times[0,1)} d x d y \bar{\phi} \phi^{\prime} .
$$

The prequantization map $\mathcal{P}_{N}$ is given by

$$
\mathcal{P}_{N}(f) \phi=f \phi-\frac{i}{2 \pi N}\left(\frac{\partial f}{\partial x}\left(\frac{\partial \phi}{\partial y}-2 \pi N i x \phi\right)-\frac{\partial f}{\partial y} \frac{\partial \phi}{\partial x}\right), \quad \forall f \in C^{\infty}\left(T^{2}\right) .
$$

In the $N=1$ case (the one considered in Ref. 2 and Ref. 10), this representation can also be realized on $L^{2}(\mathbb{R})$, via the isomorphism $W$ (defined between the dense subsets $\Gamma_{1}$ and $\mathcal{S}(\mathbb{R})$, the Schwartz space) given by

$$
(W \psi)(x, y)=\sum_{k=-\infty}^{+\infty} \psi(x+k) e^{-2 \pi i k y}
$$




$$
\left(W^{-1} \phi\right)(x)=\int_{0}^{1} d y \phi(x, y), \quad \phi \in \Gamma_{1}, \psi \in \mathcal{S}(\mathbb{R})
$$

Under this transformation the operators $\left(\frac{\partial}{\partial y}-2 \pi i x\right)$ and $\frac{\partial}{\partial x}$ go over to the operators $-2 \pi i x$ and $\frac{d}{d x}$, respectively.

For the $N=1$ case, Gotay considers the most natural basic set of functions which globally separates points, namely the set

$$
\{\sin (2 \pi x), \cos (2 \pi x), \sin (2 \pi y), \cos (2 \pi y)\}
$$

and then show that it is irreducibly represented. One is, thus, faced with a full quantization of the torus, by Gotay et al. criteria. This situation is presented as a genuine "go theorem", and used in Ref. 2 to help formulating the hypothesis of a possible general "no-go theorem".

We believe, however, that this representation of the Poisson algebra of the torus cannot be considered a physically meaningful quantization of the torus. Consider, for instance, the quantum operators representing functions depending on $x$ only, in the $L^{2}(\mathbb{R})$ representation:

$$
\left(\mathcal{P}_{1}(f) \psi\right)(x)=\left(f(x)-x \frac{d}{d x} f(x)\right) \psi(x) .
$$

The form of the operators clearly shows that they are unbounded, having as spectrum the whole real line. This drastic enlargement of the spectrum of classically bounded observables has, of course, dramatic consequences: for instance, the quantum measurement of the mean value of $\sin (2 \pi x)$ (or $\sin ^{2}(2 \pi x)$ ) can produce any real value. The operator $\left(\mathcal{P}_{1}(\sin (2 \pi x))\right)^{2}$ is positive of course, although not bounded, but the quantum observable $\mathcal{P}_{1}\left(\sin ^{2}(2 \pi x)\right)$ is neither. This is an example of a general fact, which is the disappearance at the quantum level of relations among pairs of commuting classical observables $f(x), g(x)$. In particular, for the above mentioned operators one gets:

$$
\left(\mathcal{P}_{1}(\sin (2 \pi x))\right)^{2}-\mathcal{P}_{1}\left(\sin ^{2}(2 \pi x)\right)=4 \pi x^{2} \cos ^{2}(2 \pi x),
$$

where it is clear that the multiplicative operator appearing in the right hand side does not correspond to the quantization of any observable on the torus. If this representation were to be physically realizable, one could not infer the observable values of $\mathcal{P}_{1}\left(\sin ^{2}(2 \pi x)\right)$ just by measuring $\mathcal{P}_{1}(\sin (2 \pi x))$.

Among the broken classical relations one finds also the trigonometric one, which appears strongly violated at the quantum level:

$$
\left(\mathcal{P}_{1}(\sin (2 \pi x))\right)^{2}+\left(\mathcal{P}_{1}(\cos (2 \pi x))\right)^{2}=1+4 \pi^{2} x^{2},
$$

where the unobservable operator $x$ again appears. Therefore, and although no new local degrees of freedom entered the quantum theory, we find no traces of the global aspects of the phase space one has begun with. 


\section{Discussion}

It is clear from the unboundedness of the spectrum of the quantum operators mentioned in Sec. 2 and the related violation of the trigonometric relation that information about the global topology of the phase space $T^{2}$ was lost. This representation effectively "decompactifies" the phase space. This "decompactification" accounts for the infinite dimensionality of the Hilbert space, which goes against the physical expectation of having only a finite number of independent quantum states associated with a compact phase space.

In our opinion, this example shows that irreducibility by itself does not guarantee, in a general symplectic manifold, that one gets a physically acceptable quantum theory. In globally non-trivial cases functional relations among basic observables appear as essential, since they carry information about the global topology of the phase space. In the present case of the torus we showed that crucial global topological information was indeed lost in the quantization process, although the irreducibility requirement was satisfied.

It is worth mentioning that the Dirac-like approach based on the strict "Poisson bracket goes to commutator" rule does not look appropriate in the torus case. Contrary to other known cases, e.g. cotangent bundles and $S^{2}$, the Poisson algebra of the torus does not seem to admit subalgebras that separate points (and contains the constant function 1), other than $C^{\infty}\left(T^{2}\right)$ itself (and dense subalgebras, e.g the one generated by the basic set $(10)$ and the constant function 1 , which is made of functions having a finite Fourier decomposition). In particular, it is known that no such finite-dimensional subalgebra can be found 11 Thus, regarding question (a) in the introduction, it seems that for $T^{2}$ one is bound to impose the Dirac quantum condition on the whole Poisson algebra, like Gotay et al. did. But it was recently proved that no non-trivial finite-dimensional Lie representation of $C^{\infty}(\mathcal{M})$

can be found, for any connected compact symplectic manifold $\mathcal{M} .22$ Also, it is an established fact that for compact $\mathcal{M}$ every non-trivial prequantization of $C^{\infty}(\mathcal{M})$ in a infinite dimensional space includes unbounded operators. 13 Thus, it seems that, just like in Gotay's proposal, every conceivable Dirac-like quantization of the torus will produce unbounded operators, thus leading to problems like the ones we discussed.

On the other hand it is known that a sequence of finite-dimensional Lie algebras $g(N)$ exists, such that, in the $N \rightarrow \infty$ limit a representation of the Poisson algebra $C^{\infty}\left(T^{2}\right)$ is approached. Thus, although no $g(N)$ is a representation of $C^{\infty}\left(T^{2}\right)$ (they are in factrepresentations of a deformed algebra), the correct classical limit is achieved. $14,25.16 .17$

\section{Acknowledgments}

I thank José Mourão for enlightening discussions and constant encouragement. I thank Nenad Manojlovic and Yuri Kubyshin for valuable discussions. This work was supported by FCT under grants Praxis XXI BD 3429 and CERN/S/FAE/1111/96. 


\section{References}

1. A.A. Kirillov, in Dynamical Systems IV: Symplectic Geometry and its Applications, Encyclopedia Math. Sci. IV, eds. V.I. Arnol'd and S.P. Novikov (Springer, New-York, 1990) pp. 137-172.

2. M.J. Gotay, H.B. Grundling, G.M. Tuynman, J. Nonlinear Sci. 6, 469 (1996).

3. A. Ashtekar, Lectures on Non-Perturbative Canonical Quantum Gravity (World Scientific, Singapore, 1991).

4. M.J. Gotay, H. Grundling, C.A. Hurst, Trans. Am. Math. Soc. 348, 1579 (1996).

5. M.J. Gotay, H.B. Grundling, On Quantizing $T^{*} S^{1}$, preprint quant-ph/9609025 (1996).

6. F. Ziegler, Quantum representations and the orbit method, in Méthode des orbites et representations quantiques, $\mathrm{PhD}$. Thesis (Université de Provence, 1996)

7. A. Ashtekar, R. Tate, J. Math. Phys. 35, 6434 (1994)

8. A. Ashtekar, C.J. Isham, Class. Quant. Grav. 9, 1433 (1992)

9. A. Ashtekar, J. Lewandowski, in Knots and quantum gravity, eds. J. Baez (Oxford university Press, Oxford 1994)

10. M.J. Gotay, in Quantization, Coherent States and Complex Structures, eds. J.-P. Antoine et al. (Plenum, New York, 1995) pp. 55-62.

11. M.J. Gotay, J. Grabowski, H.B. Grundling, An Obstruction to Quantizing Compact Symplectic Manifolds, preprint dg-ga/9706001 (1997)

12. V.L. Ginzburg, R. Montgomery, Geometric Quantization and No Go Theorems, preprint dg-ga/9703010 (1997).

13. A. Avez, C.R. Acad. Sci. Paris A279, 785 (1974)

14. M.A. Rieffel, Commun. Math. Phys. 122, 531 (1989)

15. D.B. Fairlie, P. Fletcher, C.K. Zachos, Phys. Lett. B218, 203 (1989)

16. M. Bordemann, E. Meinrenken, M. Schlichenmaier, Commun. Math. Phys. 165, 281 (1994)

17. V. Aldaya, M. Calixto, J. Guerrero, Commun. Math. Phys. 178, 399 (1996)

E-mail adress: jvelhi@ualg.pt 